ANNALES

UNIVERSITATIS MARIAE CURIE-SKŁODOWSKA LUBLIN - POLONIA

VOL. LXXII, 2 SECTIO AA

\title{
Thermal and spectroscopic studies of polymer protective coatings used in optical fibers technology
}

\author{
Malgorzata Gil $^{\mathrm{a},{ }^{*},}$, Malgorzata Kuklinska ${ }^{\mathrm{b}}$, Lidia Czyzewska ${ }^{\mathrm{a}}$, \\ Grzegorz Wójcik $^{\mathrm{a}}$ and Pawel Mergo ${ }^{\mathrm{a}}$ \\ ${ }^{1}$ Laboratory of Optical Fibre Technology, Maria Curie-Sklodowska University, \\ Maris Curie-Sklodowska Sq.5, 20-031, Lublin, Poland, \\ ${ }^{2}$ Polish Centre for Photonics and Fibre Optics, Rogoznica 312, \\ 36-060 Glogow Malopolski, Poland \\ "e-mail:malgorzata.gil@poczta.umcs.lublin.pl
}

\begin{abstract}
An important factor determining the quality of optical fibers is their mechanical reliability. The control of the mechanical reliability allows define the lifetime of a fiber, which was under the stress during the work. Decisive influences on the mechanical and optical properties of the optical fibers have the protective coatings. Optical fiber technology requires that the coatings were applied on them on-line, directly after they have been drawn. So far, little attention has been paid to optimizing the conditions of coatings curing. In this work, the comparison of a thermoanalytical and spectroscopic analysis of coatings that were cured in an UV oven in which the power of the UV lamps was changed will be shown. As part of the research tasks, completed mechanical strength measurements were made with a silica fiber of $0.125 \mathrm{~mm}$ diameter.
\end{abstract}

Keywords: optical fibers, thermogravimetry, spectroscopy, mechanical strength, polymer

\section{INTRODUCTION}

An optical fiber is a flexible and optically transparent fiber made from glassy or polymer materials. Optical fibers are often used to transmit 
light in fiber-optic communications, where they permit transmission over long distances [1-3]. Specially designed fibers are also used for a variety of other applications, they can be applied in sensors or lasers [4-9]. Despite the great interest in active optical fibers, used for special purposes, still a lot of attention is paid to classical optical fibers. Currently, studies concentrate on searching for new materials from which protective coatings can be made and on optimizing the coatings curing conditions [10-13].

Optical fibers typically include a transparent core surrounded by a transparent cladding material with a lower refractive index. Therefore, light is kept in the core by the total internal reflection.

The cladding is coated by protective coatings that protect it from moisture and physical damage. These coatings are typically UV-cured urethane acrylate composite or polyimide materials applied to the outside of the fiber during the drawing process. In optical fiber drawing process typically a dual-layer coatings are employed. An inner primary coating is designed to minimize attenuation caused by microbending. An outer secondary coating protects the primary coating against mechanical damages. Sometimes for special optical fibers, a one layer of medium coatings is applied. This coating is typically mixture of primary and secondary coatings.

The presented studies were focused on the optimization of the medium coating curing process and thus verified whether and how it affects the quality of the obtained coating and thus the strength of the produced optical fibers.

\section{EXPERIMENTAL}

The typical commercially available protective coatings for telecommunication optical fibers were used. The chemical composition of the tested coatings includes: active solvent, adhesion promoter, a photoinitiator and main substance, which are urethane acrylates. Information on the thermal properties of urethane acrylates compounds can be found $[14,15]$. In addition, many additives that are a trade secret also can be present. In our investigations, mixture of DeSolite ${ }^{\circledR}$ 950-101

(primary coating, soft coating) and DeSolite ${ }^{\circledR}$ 950-075 (secondary coating, hard coating) in a weight ratio of 1:1 (medium coating) was used. In our investigations, a photopolymerization reaction was carried out in UV-oven in room temperature during drawing the silica fiber of 0.125 
$\mathrm{mm}$ diameter. The UV-oven was equipped with four commercially available vacuum-sealed medium pressure mercury lamps ( $800 \mathrm{~W}$ electric power each). The irradiated samples were distanced $10 \mathrm{~cm}$ from each lamp (Fig. 1). The thickness of obtained coatings was $0,05 \mathrm{~mm}$.

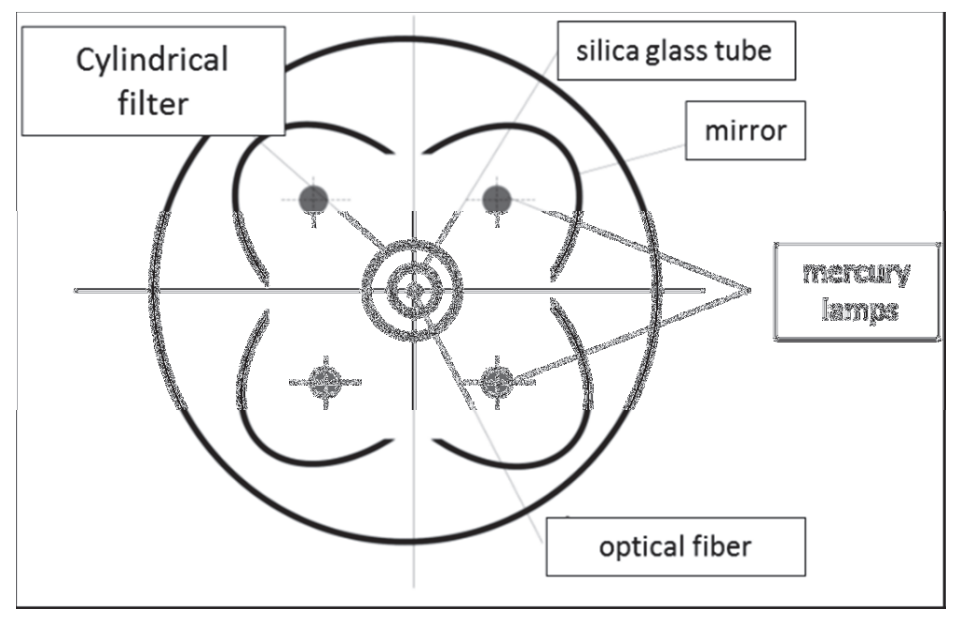

Fig. 1. The scheme of the furnace.

We used two fiber drawing speed: $30 \mathrm{~m} / \mathrm{min}$ as a typical microstructural optical fiber drawing speed and $70 \mathrm{~m} / \mathrm{min}$ to see how increased speed rate will affect the quality of the polymer coating (Tab. 1).

Thermal analysis was carried out on a STA 449 Jupiter F1, Netzsch (Selb, Germany) under the following operational conditions: heating rate $10{ }^{\circ} \mathrm{C} \mathrm{min}^{-1}$, a dynamic atmosphere of helium $\left(50 \mathrm{~cm}^{3} / \mathrm{min}\right)$ in the temperature range of $20-600^{\circ} \mathrm{C}$, sample mass of about $5 \mathrm{mg}$, sensor thermocouple type S TG-DSC. As a reference, empty Al crucible was used.

Table 1. Sample characterization.

\begin{tabular}{cccc}
\hline & Sample no. 1 & Sample no. 2 & Sample no. 3 \\
\hline $\begin{array}{c}\text { Drawing speed } \\
\text { [m/min] } \\
\text { Numbers of UV } \\
\text { lamps }\end{array}$ & 30 & 30 & 70 \\
\hline
\end{tabular}

The volatile products coming out during depolymerization and decomposition process were detected by quadrupole mass spectrometer 
QMS 403C Aëolos (Germany) coupled on-line to STA instrument. The mass spectrometer was connected on-line to STA instrument by quartz capillary heated to $300^{\circ} \mathrm{C}$. The QMS was operated with an electron impact ionizer with energy $70 \mathrm{eV}$. The measurements performed in scan mode for $\mathrm{m} / \mathrm{z}$, where $m$ is the mass of molecule and $z$ is a charge of the molecule in electron charge units in the range from 10 to $100 \mathrm{amu}$ allowed to identify all possible volatile particles produced during the decomposition.

The dynamic mechanical strength of fabricated fibers was carried out on Zwick/Roell Z2.5 testing machine. We used typical procedure used for telecommunication optical fibers. For mechanical testing $0,5 \mathrm{~m}$ long pieces of fibers were used. The tensile speed was equal to $0,05 \mathrm{~m} / \mathrm{min}$ [16-18].

\section{RESULTS AND DISCUSSION}

\subsection{Thermal and spectroscopic properties}

By using the coupled methods (TG/DSC/MS) it was possible to obtain complete characterization of phenomena occurring during the temperature increase simultaneously. Based on thermal analysis (TG) we get information about the temperature at which mass change occurs and about the quantity of this change. It is also possible to conclude about thermal stability of the analysed samples.

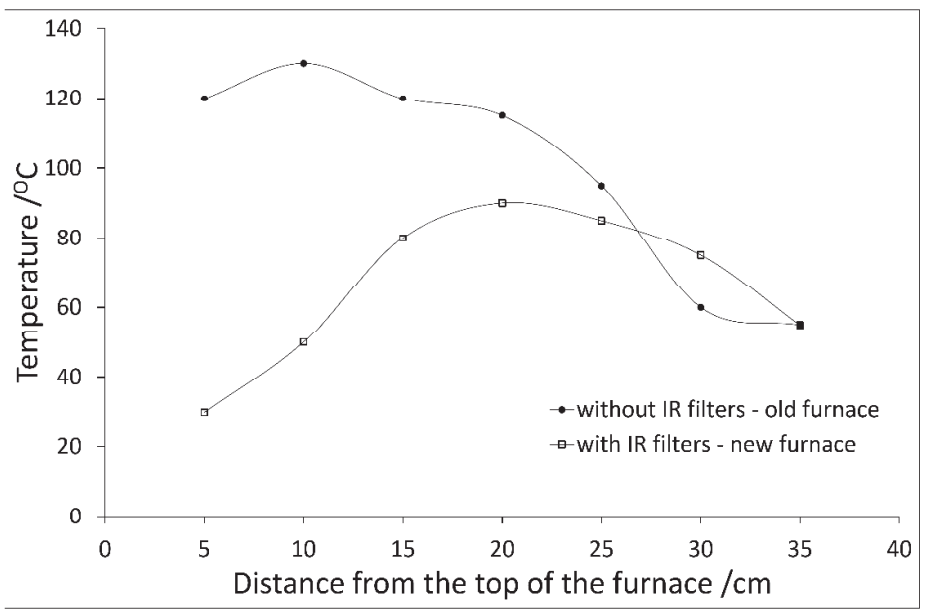

Fig. 2. Temperature distribution in UV-oven. 
The knowledge what we get from differential scanning calorimetry (DSC) concerns exo- and endothermic phenomenon arising during the analysis. Identification of gas evolved during the temperature increase by quadrupole mass spectrometer (QMS) allows predicting probable mechanism of polymers degradation and depolymerisation. Because curing procedure take place during the optical fibers drawing process, at first temperature distribution in UV-oven was tested (Fig. 2).

Next all the liquid coatings were subjected to a thermoanalytical and spectroscopic analysis (Fig. 3-8).

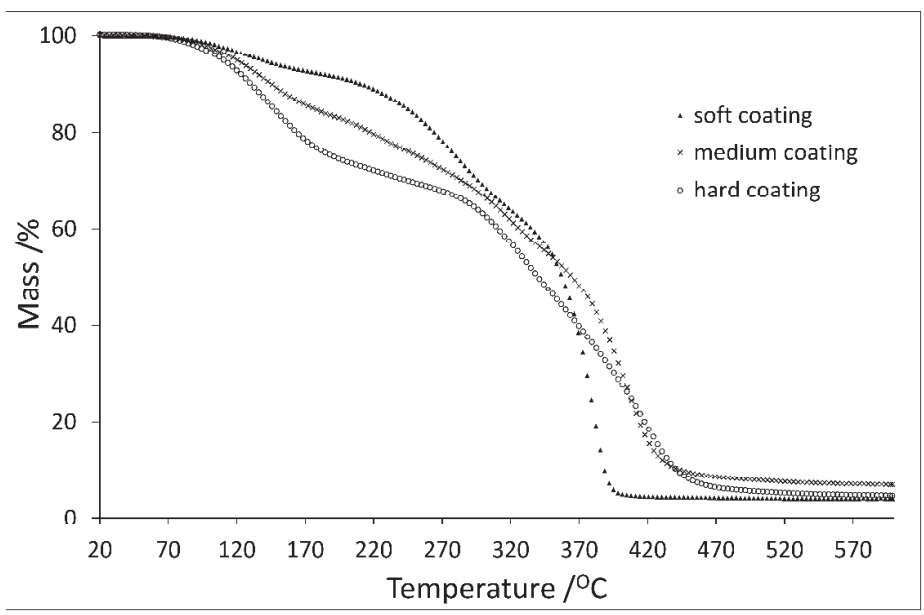

Fig. 3. TG curves for liquid samples.

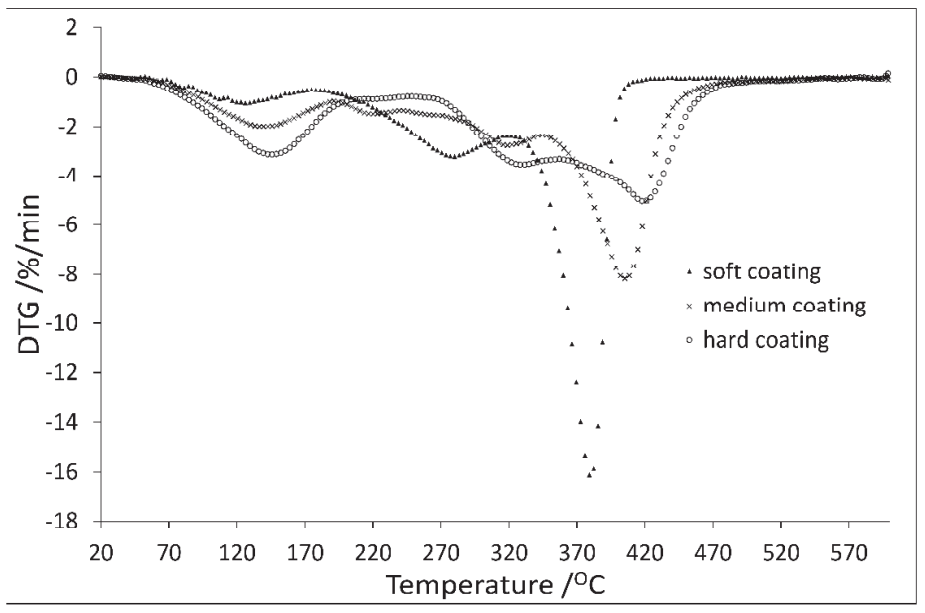

Fig. 4. DTG curves for liquid samples. 


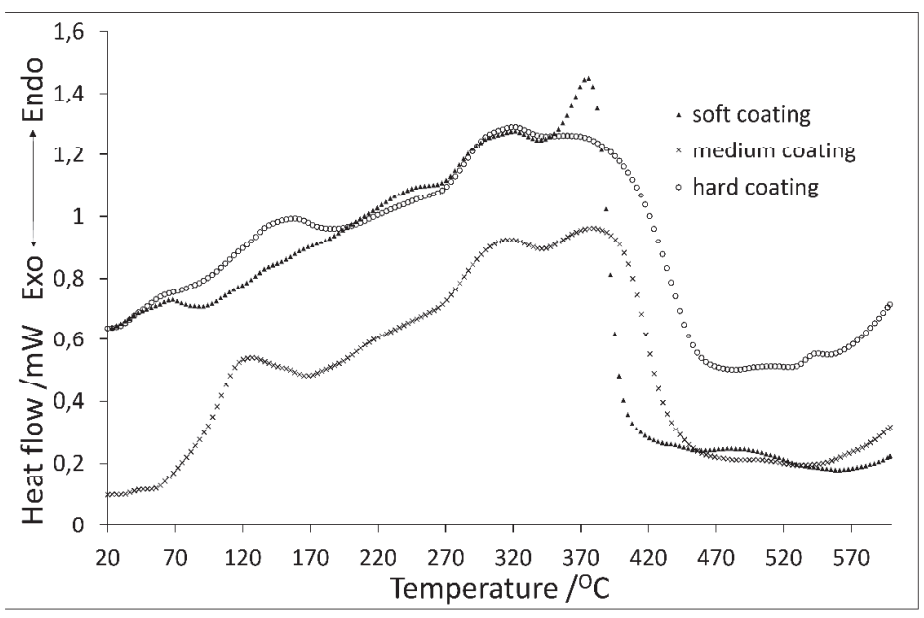

Fig. 5. DSC curves for liquid samples.

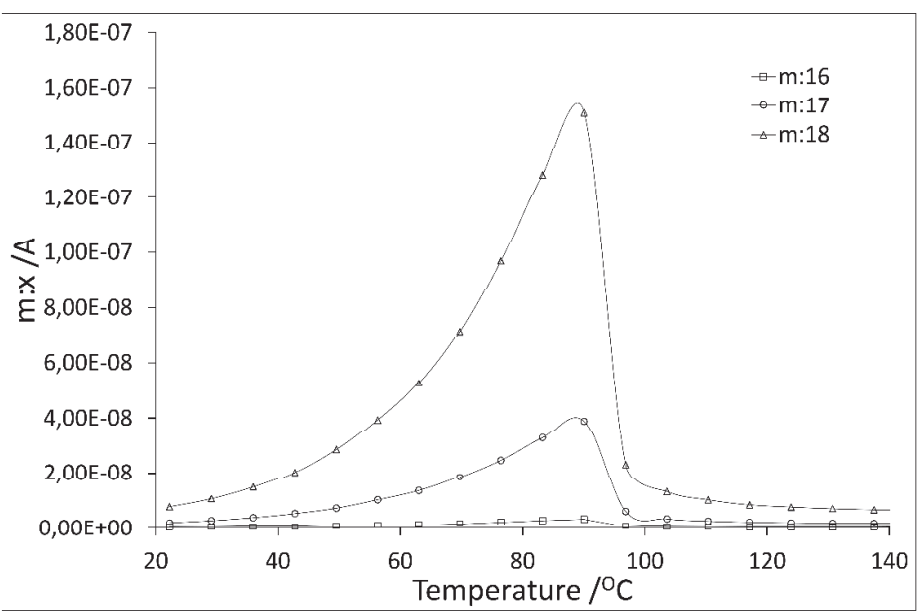

Fig. 6. Mass spectroscopy curves for soft coating.

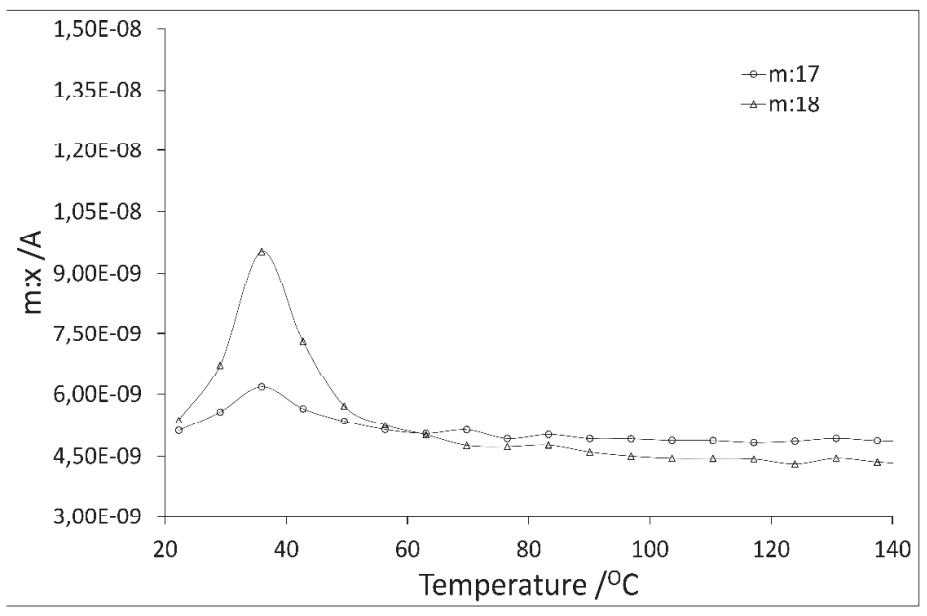

Fig. 7. Mass spectroscopy curves for medium coating. 




Fig. 8. Mass spectroscopy curves for hard coating.

Based on the results it was found that the temperature in the UV-oven is definitely too high (old furnace Fig. 2). Until the temperature of $130^{\circ} \mathrm{C}$, which is the maximum temperature in the furnace, in all analysed samples endothermic reaction was observed (Fig. 5). Spectroscopic analysis (Fig. 6-8) showed that the mass loss is associated to water evaporation, the presence of ions m:17 and 18 was observed. They appear at the temperature range $20-100^{\circ} \mathrm{C}$ for soft coating, $20-50^{\circ} \mathrm{C}$ for medium and hard coating. Furthermore, for soft and hard coating ion m:16 appears, which may indicate the presence of amidogen which can be derived from an amino group. To compare the thermal stability of the analysed liquid coatings based on TG curves the amount of the mass loss at $130^{\circ} \mathrm{C}$ and based on DTG curves the first maximum rate of mass loss was checked (Fig. 3-4, Tab. 2). DTG curves show multi-stage coatings degradation, but because our interest relates only to the phenomena occurring till $130^{\circ} \mathrm{C}$ we will not analyze other.

Table 2. Thermal stabilities of liquid samples.

\begin{tabular}{cccc}
\hline & Soft coating & $\begin{array}{c}\text { Medium } \\
\text { coating }\end{array}$ & Hard coating \\
\hline $\begin{array}{c}\text { Mass loss [wt.\%] } \\
\text { at } 130^{\circ} \mathrm{C}\end{array}$ & 4.3 & 7 & 10 \\
DTG $\max \left[{ }^{\circ} \mathrm{C}\right]$ & 125 & 140 & 146 \\
\hline
\end{tabular}


DTG curve, where a shift toward higher temperatures was observed, indicates that the highest thermal resistance has a hard coating. However, it should be noted considering the amount of mass loss that the best thermal properties has a soft coating.

At this point, it is necessary to emphasize the importance of mass loss quantity in the optical fibers technology. The temperature in which the smallest value of mass loss is record, $(0.01 \mathrm{wt} . \%)$, is our temperature limit, which during the polymer optical fiber drawing procedure should not be exceeded.

If we however cross this value, this would result in the decomposition of the coating materials, which effectively change the structure of polymer formed during photopolymerization process and causes a decrease in the mechanical strength of the optical fiber.

The obtained results forced us to redesign the UV-oven. Between the UV lamps and optical fiber IR filters have been installed. The temperature characteristic of the furnace after adding the IR filters is presented on Figure 2 (new furnace). As can be seen, the introduction of filters significantly reduces the temperature in the oven.

The next stage of the research concerned the thermal and spectroscopic analysis of cured medium coating (Fig. 9-14).

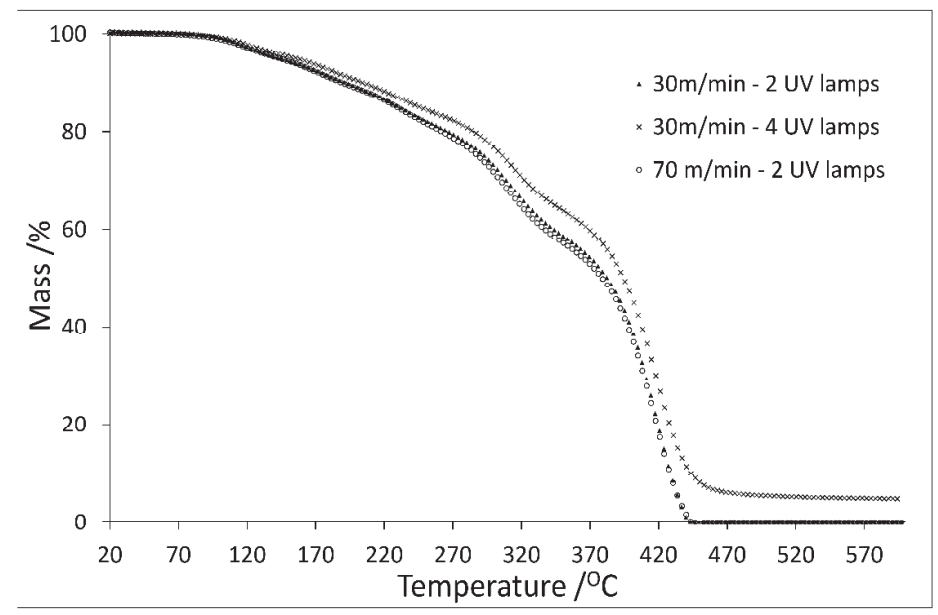

Fig. 9. TG curves for cured samples. 




Fig. 10. DTG curves for cured samples.

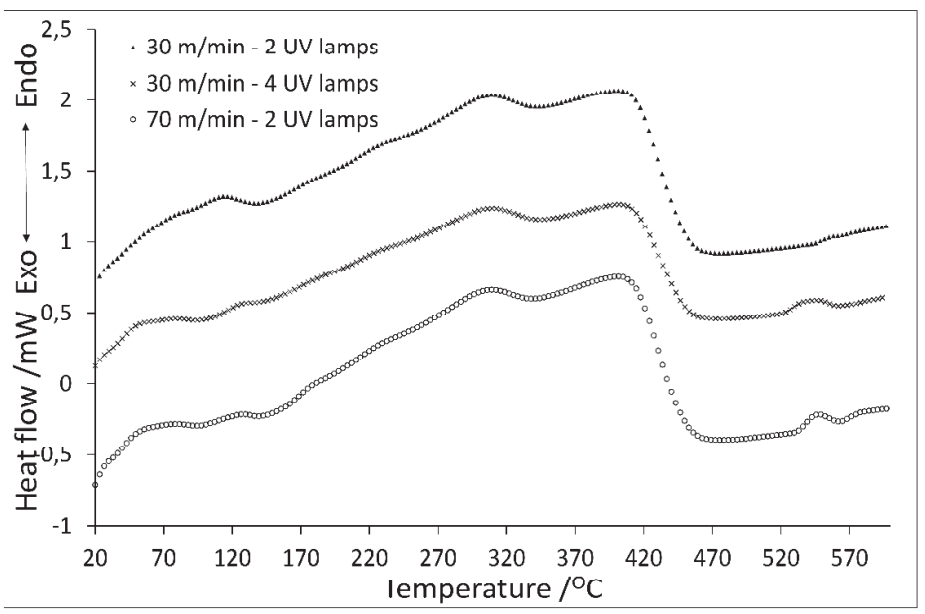

Fig. 11. DSC curves for cured samples.

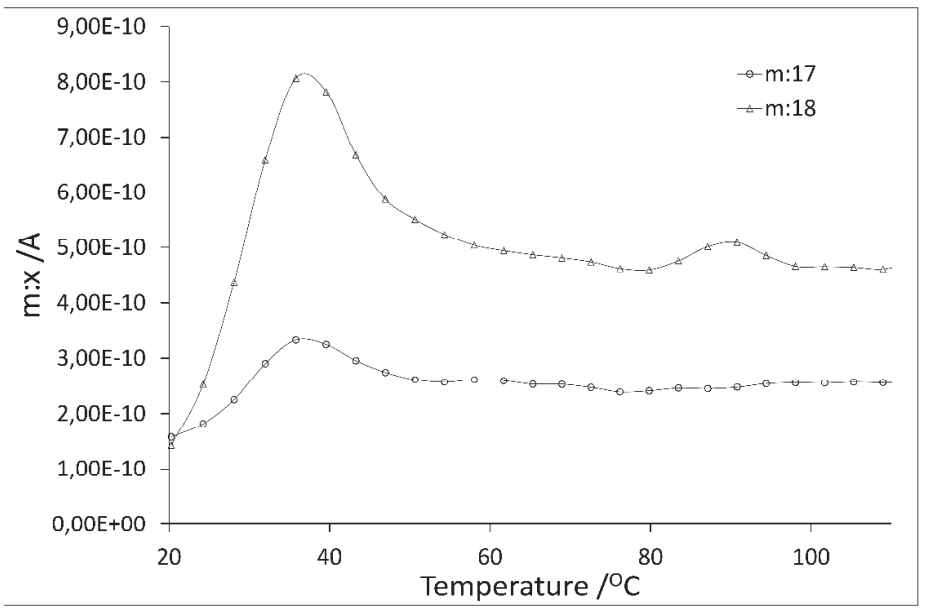

Fig. 12. Mass spectroscopy curves for sample no. 1. 


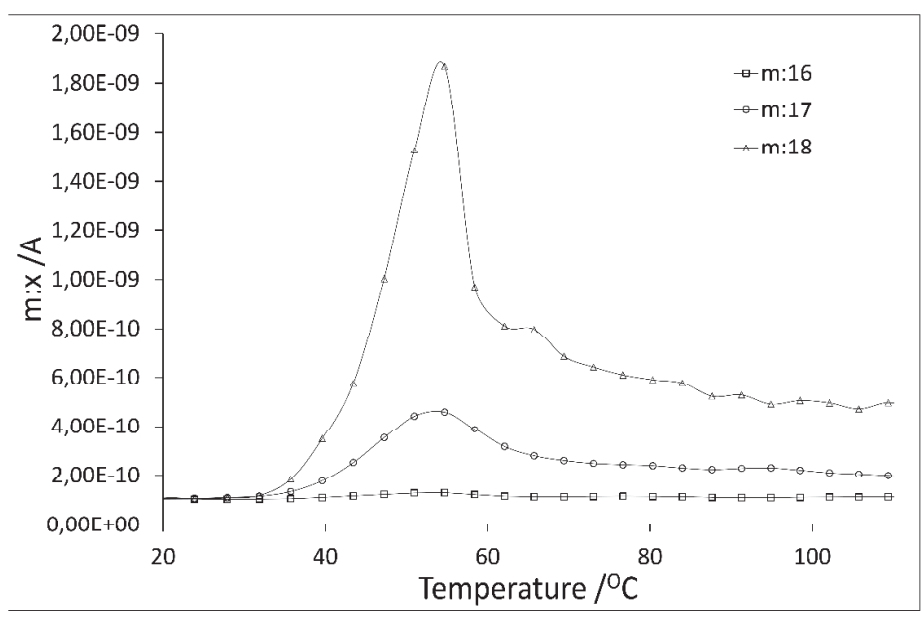

Fig.13. Mass spectroscopy curves for sample no. 2.



Fig.14. Mass spectroscopy curves for sample no. 3.

The maximum temperature after changing the furnace structure is $90^{\circ} \mathrm{C}$, so further studies will concern the changes taking place till this temperature. Based on thermal studies (Fig. 9-10) it was found that all test samples show a similar thermal stability, maximum on DTG curves are at the temperature of about $124^{\circ} \mathrm{C}$. In contrast, the sample no. 1 shows the lowest mass loss at a temperature of $90^{\circ} \mathrm{C}$ (Tab. 3).

Table 3. Thermal stabilities of cured samples.

\begin{tabular}{cccc}
\hline & Sample no. 1 & Sample no. 2 & Sample no. 3 \\
\hline $\begin{array}{c}\text { Mass loss [wt.\%] } \\
\text { at } 90^{\circ} \mathrm{C}\end{array}$ & 0.18 & 0.36 & 0.35 \\
DTG $\max \left[{ }^{\circ} \mathrm{C}\right]$ & 123 & 125 & 124 \\
\hline
\end{tabular}


On the DSC curves (Fig. 11) in the discussed temperature range endothermic processes can be observed, which indicates the evaporation of water, which are further confirmed by mass spectroscopy analysis. In all tested samples the presence of ions m:17 and 18 (Fig. 12-14) at the temperature range of $20-100^{\circ} \mathrm{C}$ for samples no. 1 , between $30^{\circ} \mathrm{C}$ and $90^{\circ} \mathrm{C}$ for sample no. 2 and for sample no. 3 between $20^{\circ} \mathrm{C}$ and $90^{\circ} \mathrm{C}$ was noted. Additionally, in the case of sample no. 2 the presence of ion m:16 in the temperature range $30-65^{\circ} \mathrm{C}$ was observed, which may indicate the presence of amidogen which can be derived from an amino group. In the case of sample no. 3 the appearance of ions m:15 (most likely derived from methyl groups) at $70^{\circ} \mathrm{C}$ and the ion m:29 (most likely derived from aldehyde groups) at a temperature of $55^{\circ} \mathrm{C}$ can be observed.

Based on the thermal and spectroscopic results it was possible to conclude that the sample no. 1 shows the best physio-chemical properties. Despite the presence of ions m:17 and 18 till $90^{\circ} \mathrm{C}$, sample no. 1 shows the smallest mass loss $(0.18 \mathrm{wt} . \%)$, and this loss is associated only to the water evaporation. In the case of sample no. 2 and 3 the presence of additional ions can be observed, which may indicate the start of thermal degradation of the coatings. Additionally, their mass loss is about two times larger than for the sample no 1.

\subsection{Mechanical properties}

For each conditions (sample 1-3, Tab. 1) the $100 \mathrm{~m}$ long optical fibers were drawn. Next, each sample was divided on $0.5 \mathrm{~m}$ pieces. For the one hundred freely selected pieces of each sample, mechanical strength was measured.

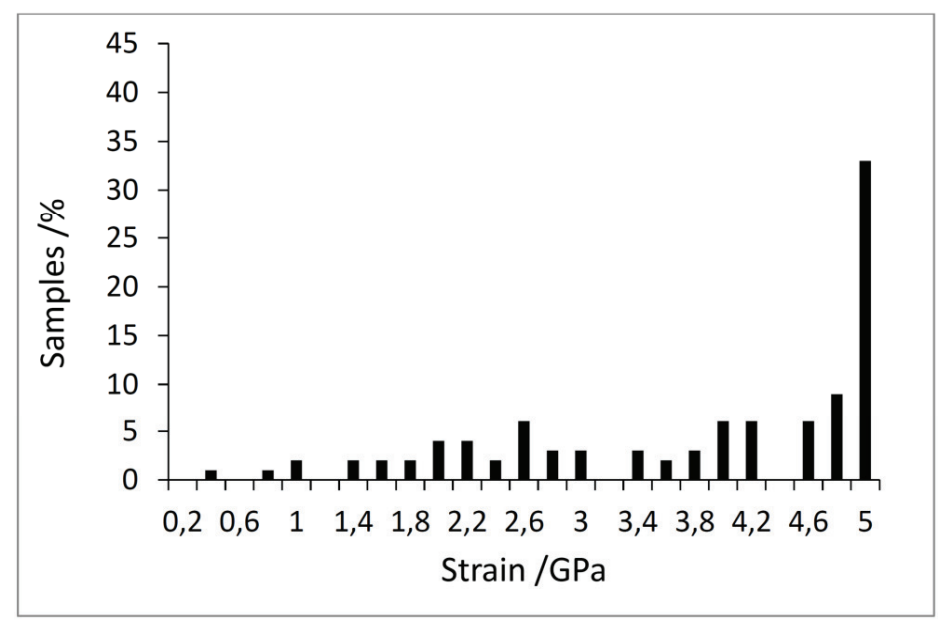

Fig. 15. Histogram for sample no. 1. 
The results are presented as histograms showing the distribution of fracture stress value that means the number of optical fiber samples breaking in defined stress ranges.

Based on mechanical tests (Fig. 15-17) it was found that sample no. 1 exhibits strength of $4.8 \pm 0,2 \mathrm{GPa}$ for more than $50 \%$ samples.



FIGURE 16 Histogram for sample no. 2

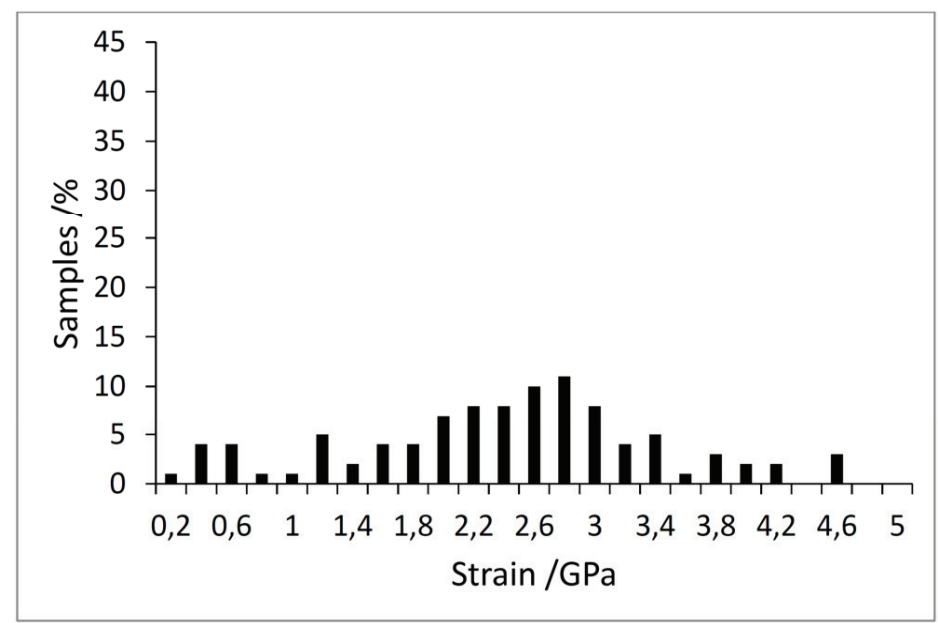

Fig. 17. Histogram for sample no. 3.

It can be observed that mechanical properties changed after increasing the number of UV lamps and for sample no. 2 the tensile strength of more than $60 \%$ samples is $4.8 \pm 0,2 \mathrm{GPa}$. On the other hand, the greatest value of mechanical strength for both samples is $5 \mathrm{GPa}$, and almost $35 \%$ of the samples exhibits this value for coating named sample no. 1 , whereas only about $18 \%$ for coating named sample no. 2 . Based on 
results obtained for sample no. 3 it can be seen that tensile strain decreases with increase in drawing speed.

\section{CONCLUSIONS}

Based on the thermoanalytical and mechanical results it was found that the optimum fiber drawing speed is $30 \mathrm{~m} \mathrm{~min}^{-1}$ with $2 \mathrm{UV}$ lamps (sample no. 1). The speed rate of $70 \mathrm{~m} \mathrm{~min}^{-1}$ is too high so it is not possible for the protective coatings to be fully cured, as indicate the high mass loss $(0.35$ wt.\%). The presence of ions m:15 and 29 that are probably the result of evaporation of uncured substances further confirms this observation. Good mechanical properties of the sample no. 2 may indicate the proper protective coating curing process, but based on thermal analysis where significant mass loss (0.36 wt. \%) can be observed, the use of $4 \mathrm{UV}$ lamps is not preferred. Additionally, the presence of ion m:16 may result from the thermal degradation of the coating.

\section{ACKNOWLEDGEMENTS}

The work was supported by the project POIG.01.03.01-06-085/12 "Advanced optical photonic structures for innovative telecommunications networks", financed by the European funds, the Polish national measures and entrepreneur's own funds.

\section{REFERENCES}

[1] J. M. Senior, M. Y Jamro. Optical fiber communications: principles and practice. third ed. Prentice Hall, 2009.

[2] P. St. J. Russell, Photonic crystal fibers Science. 299, 358-362, (2003).

[3] H. Kubota H, K. Suzuki, S. Kawanishi, M. Kakazawa, M. Tanaka, M. Fujita, Low-loss, $2 \mathrm{~km}$-long photonic crystal fibre with zero GVD in the near IR suitable for picosecond pulse propagation at the $800 \mathrm{~nm}$ band, Postdeadline paper CPD3, Conference on Lasers and Electro-Optics CLEO 2001, Baltimore, MD, USA.

[4] M. Monro, W. Belardi, K. Furusawa, J. C. Baggett, N.G.R. Broderick, D. J. Richardson, Sensing with microstructured optical fibres, Meas. Sci. Technol., 12, 854-858, (2001). 
[5] J.M. Fini, Microstructure fibres for optical sensing in gases and liquids, Meas. Sci. Technol., 15, 1120-1128, (2004).

[6] O. Frazão O, T. Martynkien, J. M. Baptista, J. L. Santos, W. Urbanczyk, J. Wojcik, Optical refractometer based on a birefringent Bragg grating written in an H-shaped fiber, Opt. Lett., 34, 76-78, (2009).

[7] T. Martynkien, G. Statkiewicz-Barabach, J. Olszewski, J. Wojcik, P. Mergo, T. Geernaert, C. Sonnenfeld, A. Anuszkiewicz, M.K. Szczurowski, K. Tarnowski, M. Makara, K. Skorupski, J. Klimek, K. Poturaj, W. Urbanczyk, N. Nasilowski, F. Berghmans, H. Thienpont, Highly birefringent microstructured fibers with enhanced sensitivity to hydrostatic pressure, Opt. Express., 18, 15113-15121, (2010).

[8] M. Hautakorpi, M. Mattinen, H. Ludvigsen, Surface-plasmon-resonance sensor based on three-hole microstructured optical fiber, Opt. Express, 16, 8427-8432, (2008).

[9] L. Rindorfii, O. Bang, Sensitivity of photonic crystal fiber grating sensors: biosensing, refractive index, strain, and temperature sensing, JOSA B., 25, 310-324, (2008).

[10] A.A. Stolov, D.A. Simoff, J. Lie, Thermal Stability of Specialty Optical Fibers, J. Lightwave Technol., 26, 3443-3451, (2008).

[11] M. Sato, T. Ishigure, Y. Koike, Thermally stable high-bandwidth gradedindex polymer optical fiber, J. Lightwave Technol., 18, 952-958, (2000).

[12] M. Asai, Y. Yamaki, S. Takahashi, Y. Koike, High-thermally stable and high-bandwidth graded index plastic optical fiber for vehicle networks, J. Polym. Sci., Part B: Polym. Phys., 49, 1464-1469, (2011).

[13] C.S.B. Ruiz, L.D.B. Machado, J.A. Vanin, J.E. Volponi, Cure Degree Estimation of Photocurable Coatings by DSC and Differential Photocalorimetry, J. Therm. Anal. Calorim., 67, 335-341, (2002).

[14] L.J. Chen, Q.L. Tai, L. Song, W.Y. Xing, G.X. Jie, Y. Hu, Thermal properties and flame retardancy of an ether-type UV-cured polyurethane coating, Express Polym Lett., 4, 539-550, (2010).

[15] A.A. Alshuiref, H.G. Ibrahim, A. Abduallah, M.A. Edali, Thermal and Mechanical Analysis of Urethane Acrylate Graft Copolymer Part A: Urethane Macromonomer Base on TDI and EG, Int. J. Chem., 5, 58-79, (2013).

[16] M.A. Matusewicz, J.Wojcik, P. Mergo, A. Walewski, Mechanical strength of photonic crystal fibres, Photonics Lett Pol., 2, 19-21, (2010).

[17] M. Gil, G. Wojcik, K. Szewczak, J. Pedzisz, J. Kopec, L. Czyzewska, A. Walewski, A. Gorgol, W. Podkoscielny, P. Mergo, Influence of X-rays on the thermal properties of poly(methyl methacrylate), Photonics Lett Pol., 6, 151-153, (2014).

[18] TIA/EIA-455-28-B FOTP-28 - Measuring Dynamic Strength and Fatigue Parameters of Optical Fibers by Tension (superceded by TIA/EIA-455-28-C). 